Tecnología e Innovación Tecnologia e Inovação Tecnology and Innovation
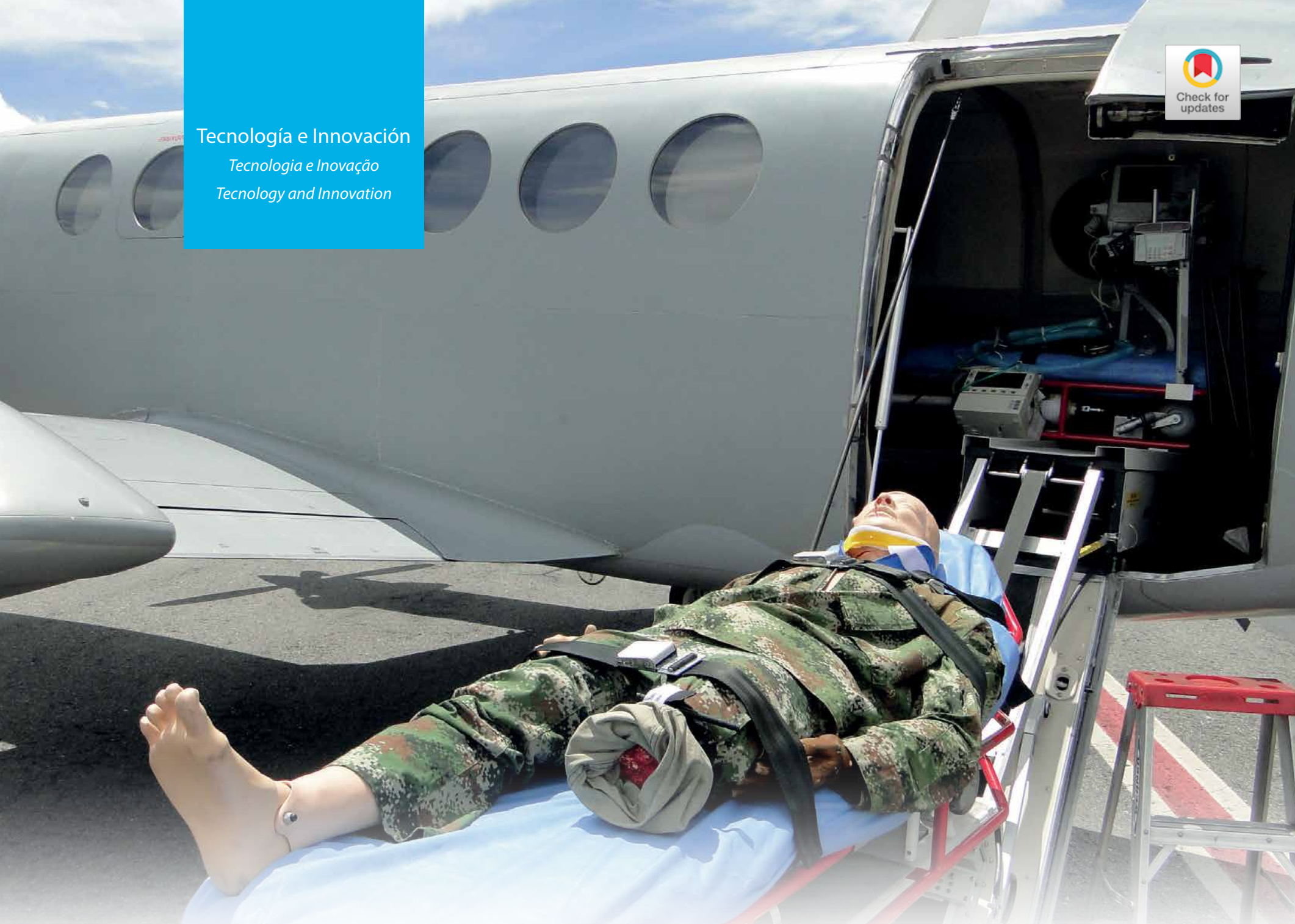

\title{
APLICACIÓN DE LA BIOTELEMETRÍA PARA TRES SIGNOS VITALES ${ }^{1}$
}

APLICAÇÃO DA BIO-TELEMETRIA A TRÊS SINAIS VITAIS ${ }^{2}$

Biotelemetry Applications for Three Vital Signals ${ }^{3}$

Leonardo Juan Ramírez López ${ }^{4}$, Andrés Fernando Marín López ${ }^{5}$ Yuli Paola Cifuentes Sanabria ${ }^{6}$ Universidad Militar Nueva Granada

\section{CIENCIA Y PODER AÉREO}

ISSN 1909-7050 / E- ISSN 2389-2468 / Volumen 10 / Enero-Diciembre de 2015/ Colombia/ Pp. 1-260 Recibido: 28/11/2014

Aprobado evaluador interno: 11/12/2014

Aprobado evaluador externo: 15/12/2015

Doi: http://dx.doi.org/10.18667/cienciaypoderaereo.428 
CIENCIA Y PODER AÉREO | Revista Científica de la Escuela de Postgrados de la Fuerza Aérea Colombiana | Vol. 10 | Enero - Diciembre de 2015

\section{Para citar este artículo:}

Ramírez, L., Marín A., y Cifuentes Y. (2015). Aplicación de la biotelemetría para tres signos vitales. Ciencia y Poder Aéreo, 10 (1), 179-186. Doi: http://dx.doi.org/10.18667/ cienciaypoderaereo.428

\begin{abstract}
'Artículo científico original, que recoge los resultados de un sistema de biotelemetría aplicado a bioseñales, de la línea de investigación de Telemetría del grupo de Investigación en Telemedicina de la Universidad Militar Nueva Granada -TIGUM-, financiado por la Universidad Militar Nueva Granada PIC-ING-1667.
\end{abstract}

${ }^{2}$ Artigo científico original, que reúne os resultados de um sistema de bio-telemetria aplicado a biosinais do grupo de pesquisa Telemetria Research em Telemedicina da Universidade Militar Nova Granada-TIGUM financiado pela Universidade Militar Nova Granada PIC-ING -1667.

${ }^{3}$ Original scientific paper, reflecting the outcome of a biotelemetric system applied to biosignals of the research group Telemetry Research in Telemedicine -TIGUM- Nueva Granada Military University, funded by Nueva Granada Military University PIC-ING -1667.

${ }^{4}$ Ingeniero Electrónico, Magíster en Ing. Sistemas y Doctor en Ing. Biomédica. Líder del Grupo de Investigación en Telemedicina de la UMNG - TIGUM, Bogotá, Colombia. Correo electrónico: tigum@unimilitar.edu.co

${ }^{5}$ Estudiante de Ingeniería en Telecomunicaciones de la UMNG. Semillero Mizar del Grupo de Investigación en Telemedicina de la UMNG - TIGUM, Bogotá, Colombia. Correo electrónico: tigum@unimilitar.edu.co

${ }^{6}$ Ingeniera Industrial, Joven investigadora del Grupo de Investigación GISSIC de la UMNG, Bogotá, Colombia. Correo electrónico: cifuentes.yuli@gmail.com
Resumen: actualmente las tecnologías de la información y las comunicaciones han permitido el diseño de complejos sistemas de transmisión, recepción y análisis de señales de origen biológico. Este tipo de sistemas se caracterizan por monitorizar las señales biológicas y establecer un control continuo de los diferentes parámetros fisiológicos en los pacientes, además de contribuir a la prevención de enfermedades. Los sistemas de biotelemetría debido a su complejidad y costo tienen la desventaja de ser poco asequibles por poblaciones en lugares apartados a las entidades de salud, necesidad que ha conllevado a los investigadores del grupo de investigación TIGUM a desarrollar una arquitectura de biotelemetría operativa y funcional a corto alcance, basada en plataforma Arduino ${ }^{\oplus}$ Mega ADK., útil para el diagnóstico y prevención de enfermedades mediante el registro y trazabilidad de la información de tres signos vitales. La arquitectura utiliza las tecnologías Bluetooth y ZigBee para facilitar la trasmisión y la visualización de las señales en diferentes equipos electrónicos.

Palabras clave: arduino ${ }^{\oplus}$ mega ADK; biotelemetría; bluetooth; signos vitales.

Resumo: atualmente, as tecnologias da informação e as comunicações têm permitido o desenho de sistemas complexos de transmissão, recepção e análise de sinais de origem biológico. Tais sistemas caracterizam-se por monitorar sinais biológicas e estabelecer um monitoramento contínuo dos diferentes parâmetros fisiológicos em pacientes, além de contribuir na prevenção de doenças. Os sistemas bio-telemétricos por causa da sua complexidade e custo têm a desvantagem de não ser muito acessíveis para pessoas em áreas remotas das entidades de saúde, necessidade que levou aos pesquisadores do grupo de pesquisa TIGUM a desenvolver uma arquitetura de bio-telemetria operacional e funcional a curto alcance baseada na plataforma Arduino ${ }^{\oplus}$ Mega ADK, útil para o diagnóstico e prevenção de doenças através do registro e a rastreabilidade da informação de três sinais vitais. A arquitetura utiliza as tecnologias Bluetooth e ZigBee para facilitar a transmissão e a exibição de sinais em diferentes equipamentos eletrônicos.

Palavras-chave: arduino ${ }^{\oplus}$ mega ADK; bio-telemetria; bluetooth; sinais vitais.

Abstract: Information and communication technologies recently have allowed the complex design of transmission system, and the reception and analysis of biological signal. The monitoring of biological signals and the establishment of a continuous monitoring of different physiological parameters of the patient and the prevention of diseases characterize these kinds of systems. The biotelemetry system due to its complexity and cost has the disadvantage of not being affordable for communities isolated from health services. This reason has caught the attention of researchers of the group TIGUM, who have developed biotelemetry architecture that is functional and operational at short-range. Using Arduino ${ }^{\oplus}$ Mega ADK platform, useful for the diagnosis and prevention of diseases through the data registration and traceability of three vital signs. The architecture uses Bluetooth and ZigBee technologies to facilitate the transmission and the visualization of signals in different electronic devices.

Key Words: Arduino ${ }^{\oplus}$ Mega ADK; Biotelemetry; Bluetooth; Vital Signs. 


\section{Introducción}

La telemetría se ha convertido en una poderosa herramienta para medir, monitorear y controlar remotamente diferentes variables con resultados en tiempo real. Esta tecnología ha facilitado el transporte de datos adquiridos por un dispositivo a una estación distante para ser desplegados, guardados y analizados por personal especializado (Dodge, 2012). Dentro de las aplicaciones más comunes de la telemetría se encuentra la supervisión de distribución de recursos en la naturaleza, el control atmosférico, registro de condiciones meteorológicas, la observación de fenómenos espaciales y el uso en la biomedicina, para la obtención de datos fundamentales del funcionamiento de órganos del cuerpo humano (IBM, 2015).

Así, la biotelemetría es el sistema teledetección y transmisión de señales biológicas, entendiendo como señal biológica un tipo de señal que se genera por los diferentes procesos biológicos del ser humano, como lo son los procesos hormonales, electroquímicos, mecánicos o bioquímicos (Carrión Pérez, Ródenas García, y Rieta Ibáñez, 2007). Los sistemas biotelemétricos tiene grandes ventajas en el diagnóstico médico y en la vigilancia del paciente, ya que facilita la trasmisión de datos telemétricos evitando conexiones directas a equipo de monitoreo, que muchas veces genera incomodidad en el paciente y restricción de movimiento (Kline, 2012). Los sistemas biotelemétricos se utilizan con mayor frecuencia para el monitoreo de pacientes con enfermadades como diabetes o parecimientos cardiacos y en algunos casos en neurociencia para monitorear la actividad neuronal. Habitualmente este tipo de sistemas telemétricos se encuentran en salas de vigilancia cardiaca o de neonatología. Aunque en la actualidad y con el avance tecnológico se han desarrollado nuevas opciones biotelemétricas que pueden ser utilizadas por fuera de las instalaciones hospitalarias para realizar diagnóstico, seguimiento y monitorización de variables clínicas (Pérez Rodríguez, Brito Zurita, Sistos Navarro, Benítez Aréchiga, Sarmiento Salazar, y Vargas Lizárraga, 2015).

Dentro de las variables clínicas que frecuentemente se monitorean se encuentran los signos vitales de: frecuencia cardiaca, frecuencia respiratoria, temperatura y presión arterial. Estas mediciones básicas determinan el funcionamiento de los organos vitales del cuerpo y detectan o monitorizar problemas de salud (Jiménez Molina, Torralbas Ortega, y Rumí Belmonte, 2014). En el caso de la frecuencia cardiaca se analiza la actividad eléctrica del corazón, mediante el registro gráfico del electrocardiograma (ECG). Para el analisis de la frecuencia respiratoria se miden indirectamente la saturación de oxígeno de la sangre del paciente, generalmente se realiza mediante el uso de un pulsioximetro. Con respecto a la temperatura corporal, esta determina el equilibrio entre la producción de calor por el cuerpo y su pérdida; su continuo monitoreo contribuye a establecer un indicador de posibles complicaciones sistemicas. Para el análisis de la fuerza que ejerce la sangre contra las paredes de las arterias, conocida como presión arterial se analiza el ritmo cardíaco o el pulso, utilizando un tensiómetro (The University of Chicago Medicine, 2015).

En el campo de la investigación científica constantemente se están desarrollando sistemas biotelemétricos ambulatorios para realizar monitorización de variables clínicas que prolongar la supervivencia del paciente, disminuyan las visitas a urgencias y el costo de atención de salud. Este tipo de sistemas, tienen una metodologia individualizada, no invasiva, que ofrece la ventaja de proporcionar al paciente un mayor grado de control y fomentar la participación del paciente con el manejo de su enfermedad. Adicionalmente, utilizan redes inalámbricas, que van desde sistemas basados en comunicación vía Bluetooth (para corto alcance) hasta sistemas que utilizan comunicaciones vía satélite (para largo alcance).

Las tecnologías de transmisión inalámbrica con mayor uso en los dispositivos biotelemétricos de corto alcance son: Bluetooth y ZigBee. La tecnología Bluetooth busca principalmente la interoperabilidad de los sistemas y equipos con capacidades inalámbricas, para conformar una red inalámbrica personal (WPAN). Sus especificaciones se encuentran divididas en características de núcleo, perfiles, protocolos y transporte (Boccuzzi , 2007). Con respecto a la tecnología ZigBee también conocida como "HomeRF Lite" fue creada para aplicaciones de domótica, control industrial, sensores médicos y automatización de edificios, entre otros. La distancia de transmisión puede variar desde los 10 metros hasta los 75 metros dependiendo de la potencia de transmisión y del entorno (Baronti, 2007). Para que las tecnologías anteriormente mencionados trabajen bajo arquitecturas telemáticas abiertas, con un software "open source" es prescindible utilizar una arquitectura tecnológica basada en módulos arduinos inalámbricos, que no generan costos adicionales en centros de control, así como un sobrecosto en hardware y software especializado, ya que utilizan hadware flexible y de fácil uso.

Con el desarrollo de esta investigación, se pretende optimizar los servicios de biotelemetría mediante el uso una arquitectura inalámbrica móvil, para medición de signos vitales a nivel académico, desarollado en la plataforma arduino. Esta arquitectura permite la monitorización en tiempo real del estado del paciente y proporciona información para el subsecuente diagnóstico médico. La información biométrica reunida se envía de forma inalámbrica, mediante las tecnologias Bluetooth y ZigBee. Esta iniciativa apunta al mejoramiento social debido que este 
CIENCIA Y PODER AÉREO | Revista Científica de la Escuela de Postgrados de la Fuerza Aérea Colombiana | Vol. 10 | Enero - Diciembre de 2015

tipo de arquitectura es capaz de dar soporte a un monitoreo remoto de personas con alteración en su estado fisiológico, que se encuentren en zonas de difícil acceso a través del uso de telemetría.

\section{Materiales y método Materiales}

La arquitectura del sistema biotelemétrico se compone de dos sistema: el primero sistema es utilizado para la adquisición de las signos vitales (electrocardiograma [EGC], temperatura corporal y presión arterial ), y el segundo sistema para la transmisión de datos en tiempo real, utilizando tecnologías inalámbricas de corto alcance (Bluetooth y ZigBee) como se presentan a continuación:

\section{Sistema de adquisición}

Para el proceso de adquisición de las signos vitales fue necesaria la utilización electrodos, termómetro, transductor termistor NTC h2010 y un simulador paciente FLUKE Biomedical PS420. El hardware utilizado tipo "open source", correspondiente a la plataforma Arduino ${ }^{\circledR} \mathrm{MEGA}$ ADK, que tiene la arquitectura de los microcontroladores AVR de ATMEL. Esta tarjeta provee puertos de entrada/salida tanto analógicas como digitales que incluye un entorno de desarrollo para implementar código en lenguaje $\mathrm{C} / \mathrm{C}++$. (Robayo Alcocer, 2014). Ver Figura 1. Para que las lecturas de los signos vitales de ECG fueran procesadas por la plataforma Arduino ${ }^{\oplus}$ MEGA ADK fue necesario utilizar el amplificador de instrumentación AD620.

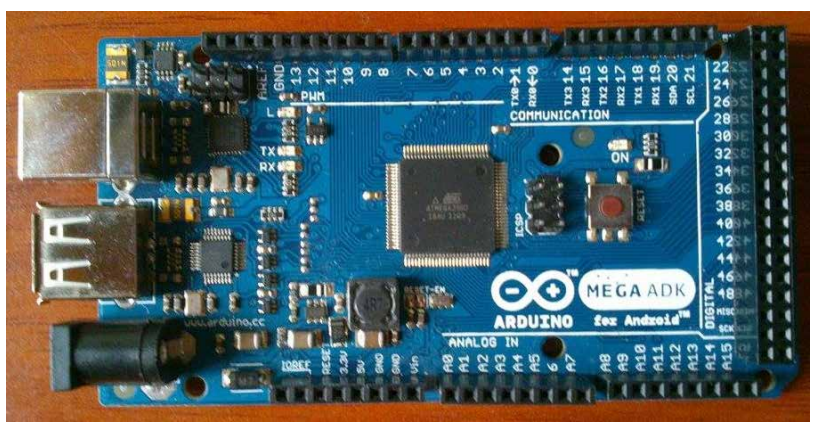

Figura 1. Arduino ${ }^{\circledR}$ Mega ADK. Fuente: autores

\section{Sistema de Transmisión}

El sistema de transmisión en una red inalámbrica permite la comunicación entre los dispositivos utilizados para la adquisición, envío, y recepción de signos vitales. Las tecnologías utilizadas en la investigación corresponden a Bluetooth y ZigBee. Las anteriores tecnologías, se comunican con la plataforma Arduino ${ }^{\circledR}$ Mega ADK mediante el módulo Shield Bluetooth HC-05 y la adaptación de la tarjeta XBee al circuito base. En la Figura 2 se muestra el componente Bluetooth instalado a la plataforma Arduino ${ }^{\oplus}$ Mega ADK.

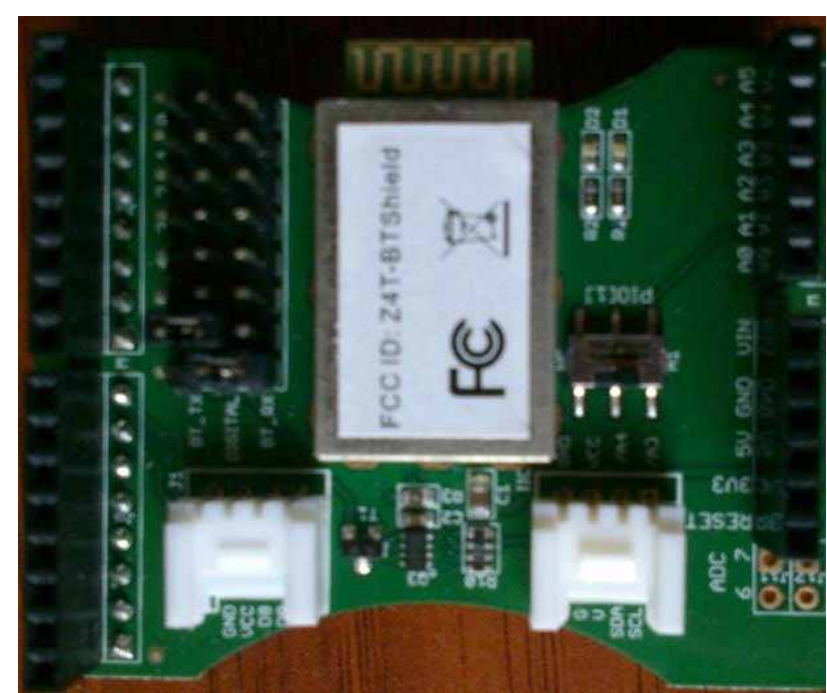

Figura 2. Bluetooth del Arduino ${ }^{\oplus}$ Mega ADK. Fuente: autores.

El módulo Bluetooth utilizado en el sistema de transmisión, corresponde a un módulo maestro- esclavo, es decir que permite realizar diferentes conexiones entre los dispositivos Bluetooth, también facilita las conexiones punto a punto para transmitir datos entre dos micro controladores o dispositivos. Es importante tener acceso al módulo mediante una interfaz serial para establecer la comunicación con el módulo y configurarlo (Geek Factory México, 2014).

El módulo Shield de XBee para Arduino, se instala directamente sobre el Arduino. Este elemento le agrega las características de comunicación inalámbrica del popular módulo XBee. Funciona con todas las series de XBee en versiones estándar y pro y para su configuración se puede realizar de manera sencilla con el software X-CTU. (5Hertz Electronica).En la Figura 3 se muestra el Shield de XBee para Arduino ${ }^{\oplus}$ Mega ADK

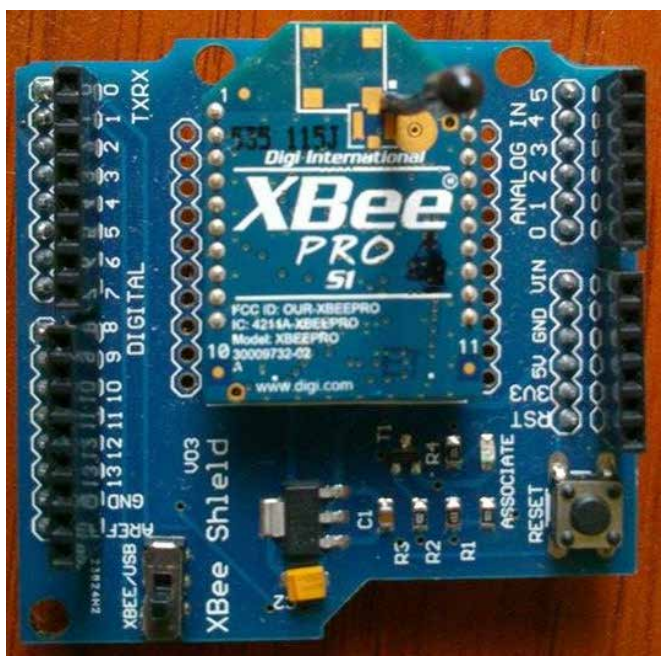

Figura 3. Shield de Xbee para Arduino ${ }^{\circledR}$ Mega ADK. Fuente: autores. 


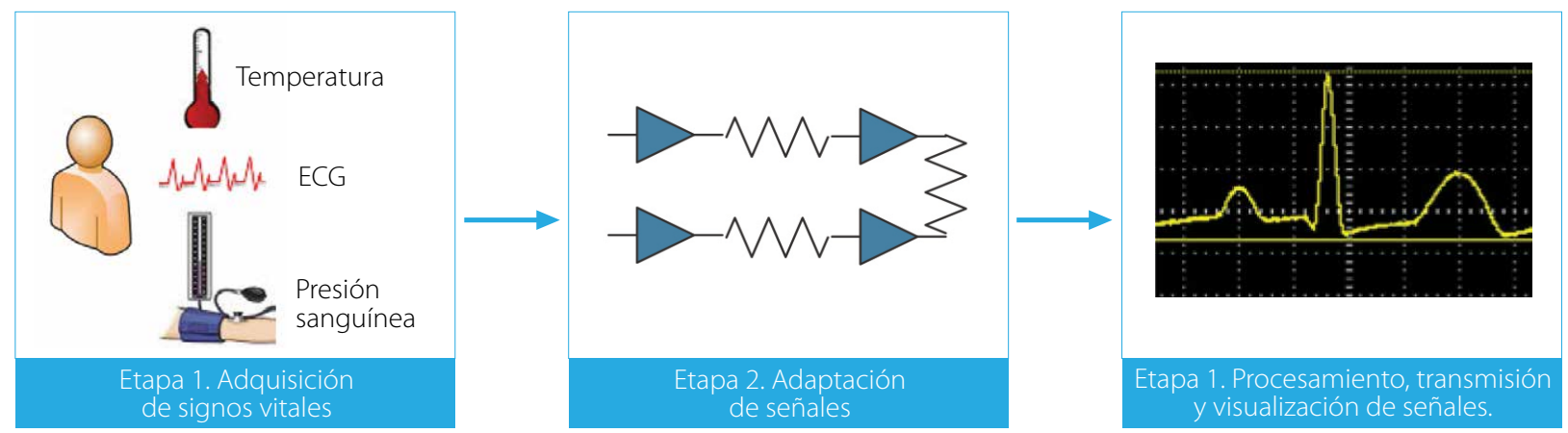

Figura 4. Diagrama de la arquitectura de biotelemetría utilizada. Fuente: elaboración de los autores.

\section{Método}

La arquitectura biotelemétrica utilizada para la medición de signos vitales se compone básicamente por tres etapas (ver Figura 4). En la primera etapa, se realiza la adquisición de los signos vitales (EGC, presión sanguínea y temperatura), posteriormente se realiza la etapa de adaptación de señales y finalmente la etapa de procesamiento, transmisión y visualización de las señales.

\section{Etapa 1: adquisición las bioseñales}

El proceso de adquisición de los signos vitales, se realizó mediante sensores que se conectan al cuerpo del paciente. En el caso del registro gráfico del electrocardiograma (ECG), se tomó un registro con los electrodos conectados al cuerpo de un voluntario, en este proceso los electrodos toman las corrientes físicas o iónicas del cuerpo y las transforman en corriente eléctrica (Melo León y Maya Quintero, 2013). Para los registros de temperatura se utilizó un transductor termistor NTC h2010, el cual en su salida entrega un voltaje en función de la temperatura corporal del paciente. Finalmente, la presión sanguínea fue obtenida mediante el simulador paciente FLUKE Biomedical PS420, debido que este simulador entrega la señal apta para ser procesada por plataforma Arduino ${ }^{\circledR}$ MEGA ADK.

\section{Etapa 2: adaptación las bioseñales}

En la etapa de adaptación de las bioseñales se amplifican las señales obtenidas en la etapa anterior, principal- mente la señal ECG, de tal forma que sea visible para su posterior manejo y/o acondicionamiento.

La señal ECG necesita ser acondicionada, debido a su baja amplitud en voltaje, que hace que sea necesario adaptarlas del tal forma que pueda ser procesada por el Arduino. (Leonardo Juan, Yohanna Patricia, y Yuli Paola, 2014). La primera etapa de adaptación las corrientes lónicas, ingresan a los trandustores y se convierten en corrientes eléctricas de bajo voltaje, posteriomente, en el amplificador la señal eléctrica se amplifica en modo diferencial y se rechaza en modo común $(A=1000)$, luego pasa por los filtros los cuales eliminan los componentes espectrales de alta y baja frecuencia, por último se elimina el ruido de la red $A C$, para obtener una señal eléctrica con una amplitud mayor a $2.5 \mathrm{~V}$ y con un ancho de banda de $0.50 \mathrm{~Hz}$ a $150 \mathrm{HZ}$ cumpliendo con regulaciones internacionales en sistemas de señales ECG (ver Figura 5).

El proceso de acondicionamiento de la temperatura se realiza utilizando un divisor de tensión entre la resistencia del termistor h2010 y una resistencia incluida en el circuito utilizado. Para determinar la temperatura del termistor, es necesario calcular el valor de las constantes A, B y C en los tres puntos $0^{\circ} \mathrm{C}, 25^{\circ} \mathrm{C}$ y $70^{\circ} \mathrm{C}$. Utilizando el modelo matemático Steinhart-Hart mediante la Ecuación 1.

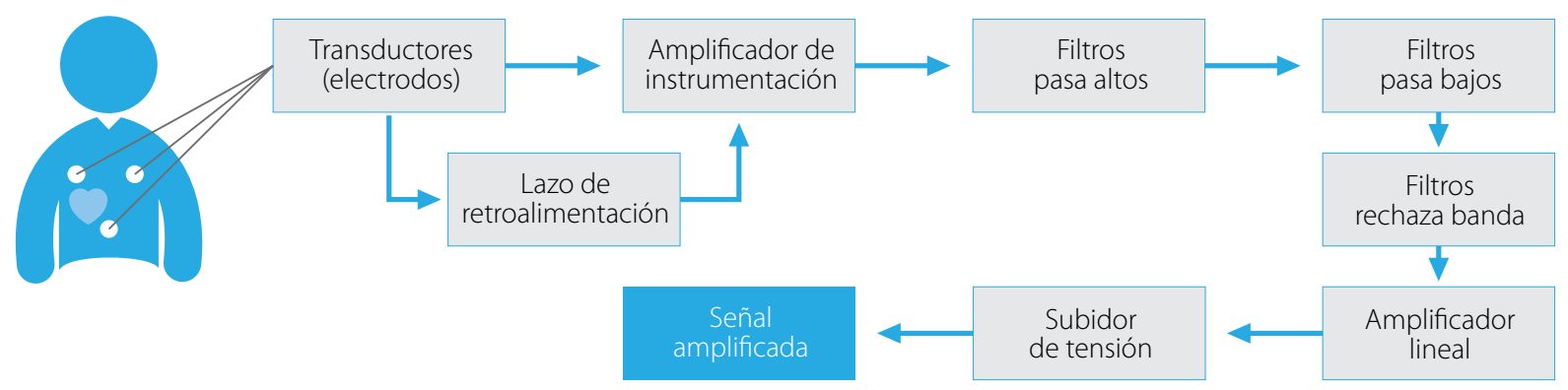

Figura 5. Diagrama de Acondicionamiento de la señal ECG. Fuente: elaboración de los autores. 
CIENCIA Y PODER AÉREO | Revista Científica de la Escuela de Postgrados de la Fuerza Aérea Colombiana |Vol. 10 | Enero - Diciembre de 2015

$$
T_{s h}=\frac{1}{A+B \cdot \log (R)+C \cdot(\log (R))^{3}}-273
$$

\section{Etapa 3: procesamiento, visualización} y envío de bioseñales

Esta etapa inicia con el procesamiento de la señal de análoga a digital a través de la tarjeta Arduino ${ }^{\circledR}$ MEGA ADK, ya que es necesario realizar esta conversión para poder visualizar las señales en la interfaz gráfica del dispositivo seleccionado.

Luego se continua con el envío de las señales utilizando las tecnología inalámbrica Bluetooth y ZigBee. Para que este proceso se ejecute adecuadamente es indispensable realizar la digitalización, la cual consiste en alistar la señal para ser trasmitida por el puerto al serial del computador. Para la visualización de la señal trasmitida mediante Bluetooth se utilizó el programa Paniri Manager V1.0 que permite la conexión entre el Bluetooth adaptado a la plataforma Arduino y el Bluetooth del computador.

En el caso de la tarjeta XBee-Pro, se configuró los dos módulos para realizar la transmisión punto a punto, el primer módulo XBee-Pro configurado, corresponde al que se encuentra adicionado a la plataforma Arduino ${ }^{\circledR}$ MEGA ADK, y el segundo módulo, se encuentra conectado al computador con sistema operativo Windows, separados a una distancia considerable.

\section{Resultados}

En la presente sección se muestran los resultados obtenidos de las pruebas realizadas para la adquisición y transmisión de los signos vitales de ECG, temperatura y presión arterial.

\section{Señal de Electrocardiograma ( ECG)}

En la adquisición de la señal de ECG, las amplitudes obtenidas de las señales fueron de 0.5, 1.0, 1.5 y $2.0 \mathrm{mV}$ con un error de exactitud de $2 \%$. El ritmo cardiacos utilizado fue

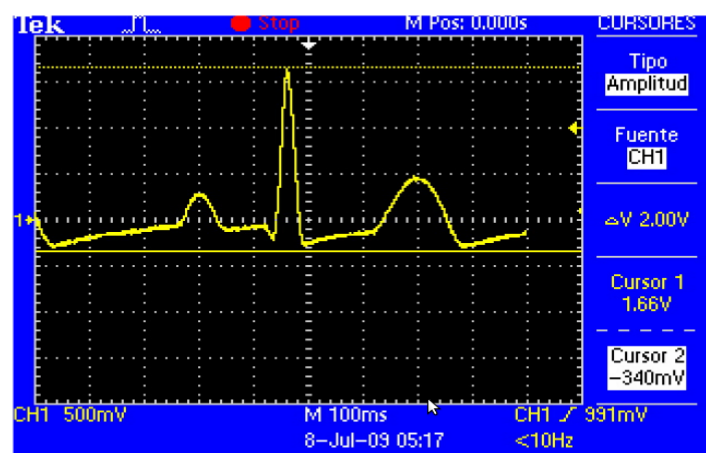

Figura 6. Adquisición de la señal EGC. entre de 30,40,60, 80, 100,120, 140, 160, 180, 200, 220, 240, 260, 280 y 300 pulsaciones por segundos (por defecto es de 80 BPM) con un error de exactitud de 1\%.

En la Figura 6 se visualiza la señal transmitida de un registro de electrocardiografía emitida desde el sistema propuesto a un osciloscopio digital. Esta visualización determina en los dos canales la comparación de la señal original enviada versus la señal recibida.

\section{Señal de temperatura}

Para la adquisición de la señal de temperatura se realizó 20 muestras con el termistor NTC h2010 y el termómetro Genetherm Clinic (elevada precisión de $+/-0.1^{\circ} \mathrm{C}$ ). Como se observa en la Tabla 1 el porcentaje de error no supera el 1\%, indicando que el registro de la señal de temperatura es adecuado, ya que un error de 1\% podría significar la diferencia entre un paciente que presente fiebre o no.

Tabla 1. Registro de temperatura corporal en voluntario

\begin{tabular}{|c|c|c|c|}
\hline$N^{\circ}$ & $\begin{array}{c}\text { Termistor } \\
\text { h2010 } \\
\end{array}$ & $\begin{array}{c}\text { Patrón } \\
\text { Termómetro } \\
\end{array}$ & $\begin{array}{c}\text { Diferencia }{ }^{\circ} \mathrm{C} \\
\text { (Sensor y } \\
\text { Patrón) }\end{array}$ \\
\hline 1 & 32,6 & 32,8 & 0,2 \\
\hline 2 & 25,1 & 25,3 & 0,2 \\
\hline 3 & 38,1 & 38 & 0,1 \\
\hline 4 & 42,3 & 43,5 & 1,2 \\
\hline 5 & 45,1 & 45 & 0,1 \\
\hline 6 & 27 & 27 & 0 \\
\hline 7 & 25,6 & 25,7 & 0,1 \\
\hline 8 & 34,5 & 34,6 & 0,1 \\
\hline 9 & 30,3 & 30,5 & 0,2 \\
\hline 10 & 44,3 & 44,5 & 0,2 \\
\hline 11 & 25,1 & 25,3 & 0,2 \\
\hline 12 & 25,6 & 25,7 & 0,1 \\
\hline 13 & 30,3 & 30,5 & 0,2 \\
\hline 14 & 38,5 & 38,7 & 0,2 \\
\hline 15 & 28,2 & 28,4 & 0,2 \\
\hline 16 & 30,3 & 30,5 & 0,2 \\
\hline 17 & 28,2 & 28,1 & 0,1 \\
\hline 18 & 28,5 & 28,6 & 0,1 \\
\hline 19 & 25,1 & 25,3 & 0,2 \\
\hline 20 & 33,2 & 33,2 & 0 \\
\hline
\end{tabular}

Fuente: elaboración de los autores. 
Tabla 2. Comparación entre Bluetooth y ZigBee para transmisión de ECG de 60 ppm con 700 muestras/s y distancia de 10m

\begin{tabular}{|c|c|c|c|c|c|c|c|}
\hline & \multicolumn{2}{|c|}{ Dispositivo } & \multicolumn{2}{|c|}{ Interfaz de usuario } & \multicolumn{3}{|c|}{ Porcentaje de error Interfaz -Dispositivo } \\
\hline $\begin{array}{c}\text { Tipo de } \\
\text { Tecnología }\end{array}$ & $\mathrm{Fd}(\mathrm{ppm})$ & $\mathrm{Ad}(\mathrm{mV})$ & Fi(ppm) & $\mathrm{Ai}(\mathrm{mV})$ & $\mathrm{Fi}-\mathrm{Fd}(\mathrm{ppm})$ & $\mathrm{Ai}-\mathrm{Ad}(\mathrm{mV})$ & $\%$ Error $((\mathrm{Ai}-\mathrm{Ad}) / \mathrm{Ad}))^{*} 100$ \\
\hline \multirow{5}{*}{ Bluethooth } & \multirow{4}{*}{60} & 0,5 & \multirow{4}{*}{60} & 0,5 & \multirow{4}{*}{0} & 0,05 & 10,0 \\
\hline & & 1 & & 1,0 & & 0,05 & 5,0 \\
\hline & & 1,5 & & 1,5 & & 0,03 & 2,0 \\
\hline & & 2 & & 2,0 & & 0,02 & 1,0 \\
\hline & & & & & $\begin{array}{c}\text { Promedio de } \\
\text { error }\end{array}$ & $4 \%$ & $4,5 \%$ \\
\hline \multirow{5}{*}{ ZigBee } & \multirow{4}{*}{60} & 0,5 & \multirow{4}{*}{60} & 0,5 & \multirow{4}{*}{0} & 0,05 & 10,0 \\
\hline & & 1 & & 1,0 & & 0,01 & 1,0 \\
\hline & & 1,5 & & 1,5 & & 0,02 & 1,3 \\
\hline & & 2 & & 2,0 & & 0,03 & 1,5 \\
\hline & & & & & $\begin{array}{c}\text { Promedio de } \\
\text { error }\end{array}$ & $3 \%$ & $3,5 \%$ \\
\hline
\end{tabular}

Fuente: elaboración de los autores.

\section{Señal de presión sanguínea}

La señal de presión emitida por el simulador paciente FLUKE Biomedical PS420 es de tipo no invasiva emitida por dos canales. La sensibilidad del transductor que tiene el simulado es de $5 \mu \mathrm{V} / \mathrm{V} / \mathrm{mmH}$ g o de $40 \mu \mathrm{V} / \mathrm{N} / \mathrm{mmHga}$ ), con una presión de $\pm 1 \%, \pm 1 \mathrm{mmHg}$. Por el canal 1 se obtuvieron señales de -10, 0, 80, 160, 240, 320 y $400 \mathrm{mmHg}$ y por el canal 2 de 10, 0, 50, 100, 150, 200 y $240 \mathrm{mmHg}$.

\section{Transmisión de señales.}

Se realizaron tres diferentes pruebas para analizar la conectividad, la calidad de la resolución en el momento de recepción de las señales transmitidas y confianza que ofrecen las tecnologías Bluetooth y ZigBee.

Con respecto a las pruebas de conectividad, se verificaron los tiempos que tarda cada una de las tecnologías en sincronizar el transmisor con el receptor. Se tomaron 40 muestras por cada tecnología midiendo el tiempo de sincronización en segundos con un cronómetro, a una distancia de $10 \mathrm{~m}$. Como resultados obtenidos se detectó que la tecnología Bluetooth tiene un promedio de $76.05 \mathrm{~s}$ en sincronizarse, con una desviación estándar de + ó-1.76 $\mathrm{s}$, mientras que la tecnología ZigBee tiene un promedio de conectividad de 256.57 s con una desviación estándar de + ó - de $39.4 \mathrm{~s}$. El anterior hallazgo, determina que la tecnología ZigBee utiliza más tiempo de sincronización para una distancia de $10 \mathrm{~m}$.

En relación a las pruebas de disponibilidad de las tecnologías, se realizaron pruebas durante un lapso de tiem- po de 90 minutos en el que se establecía la comunicación entre el dispositivo emisor y el receptor para cada una de las tecnologías, se midió el número de fallos que se presentaron en la comunicación a 10 metros. Los resultados obtenidos fueron nulos, lo que nos indica se puede pronósticar que las dos tecnologías no presentan fallas de comunicación al ser utilizadas.

La última prueba realizada corresponde a la calidad en la resolución de la imagen, para esta prueba se comparó las muestras tomadas por cada tecnología, con 700 muestras enviadas por segundo, en una distancia de 10 metros entre el dispositivo y la interfaz de usuario, los resultados obtenidos para el envió de la señal de frecuencia cardiaca de 60 ppm en cada una de las tecnologías por cada una de las tecnologías. Este tipo de ensayos fueron parcialmente desarrollados para diferentes distancias y frecuencias cardiacas (Ballesteros, Melo, y Maya Quintero, 2010). Los resultados de la comparación del uso de las tecnologías se muestran en la Tabla 2.

\section{Conclusiones}

La arquitectura para la biotelemetría implementada permitió la adquisición y transmisión de signos vitales de ECG, temperatura y presión sanguínea utilizando tecnologías inalámbricas para corto alcance y de bajo costo, como lo son las tecnologías Bluetooth y ZigBee. Los resultados obtenidos determinaron que cumplen con las características conectividad, la calidad de la resolución en el momento de recepción de las señales transmitidas y la confiabilidad. 
CIENCIA Y PODER AÉREO | Revista Científica de la Escuela de Postgrados de la Fuerza Aérea Colombiana | Vol. 10 | Enero - Diciembre de 2015

Dentro de las tecnologías evaluadas para la trasmisión de signos vitales, se utilizó un medio no guiados (transmisión inalámbrica), ya que los medios guiados son más complejos y costosos. Pero es importante aclarar que las tecnologías inalámbricas son vulnerables al ruido e interferencias electromagnéticas por esta razón se determinó que la implementación de una tecnología alámbrica en entornos con mucha contaminación electromagnética tiene mayor eficiencia en comparación con las otras tecnologías.

El uso de la plataforma Arduino ${ }^{\circledR}$ MEGA ADK facilita el procesamiento de las señales emitidas por el paciente en tiempo real. Adicionalmente, esta plataforma se caracteriza por tener un gran integración con otros dispositivos tecnológicos, los cuales al ser conectados pueden mejorar la transmisión de arquitectura biotelemetrica utilizada.

\section{Reconocimientos}

Los autores reconocen el apoyo recibido de la Vicerrectoría de Investigaciones de la Universidad Militar Nueva Granada, al proyecto PIC-ING-1667.

\section{Referencias}

Carrión Pérez, P., Ródenas García, J., y Rieta Ibáñez, J. (2007). Procesado de señales biomédicas. Univ de Castilla La Mancha.

5Hertz Electronica. (s.f.). XBee Shield para Arduino. Recuperado en diciembre de 2015. [En línea] Disponible en http://5hertz. com/index.php?main_page=product_info\&products_ $\mathrm{id}=496$

Ballesteros, D., Melo, H., y Maya Quintero, A. (2010). Sistema de transmisión inalámbrica de señales ECG y de temperatura para ambientes hospitalarios (SINHO)-Wireless transmission system of ECG and temperatura for hospital areas. Revista Ingeniería Biomédica, 4(7), 52-60.

Baronti, P. P. (2007). Wireless sensor networks: A survey on the state of the art and the 802.15.4 and ZigBee standards. Computer Communications, 30(7), 1655-1695.

Boccuzzi , J. (2007). Signal processing for wireless communications. McGraw Hill Professional.

Dodge, J. (2012). Telemetría usando redes de datos de telefonía celular. INGENIARE, 11(11), 67-78.

Geek Factory México. (febrero de 2014). Bluetooth HC-05 y HC-06 Tutorial de Configuración. Recuperado en diciembre de 2015. [En línea] Disponible en http://www.geekfactory.mx/tutoriales/bluetooth-hc-05-y-hc-06-tutorial-de-configuracion/

IBM. (2015). IBM Knowledge Center. Recuperado en diciembre de 2015, de Casos de uso de telemetría. [En línea] Disponible en http://www-01.ibm.com/support/knowledgecenter/SSFK-
SJ_8.0.0/com.ibm.mq.pro.doc/q002770_htm?lang=es

Jiménez Molina, M., Torralbas Ortega, J., y Rumí Belmonte, L. (2014). Tratado de enfermería en cuidados críticos pediátricos y neonatales. Recuperado en diciembre de 2015 de las constantes vitales, monitorización básica. [En línea] Disponible en http://www.eccpn.aibarra.org/temario/seccion1/capitulo4/ capitulo4.htm

Kline, J. (2012). Handbook of biomedical engineering. Elsevier.

Melo León, H., y Maya Quintero, A. (2013). Dispositivo para telemetría de señales biológicas que permite la utilización de diferentes tecnologías.

Pérez Rodríguez, G., Brito Zurita, O., Sistos Navarro, E., Benítez Aréchiga, Z., Sarmiento Salazar, G., y Vargas Lizárraga, J. (2015). El seguimiento telemétrico disminuye la visita a la sala de urgencia y coste de atención en enfermos con insuficiencia cardíaca crónica. Cirugía y Cirujanos, 83(4), 279-285.

Ramírez, L., Rodríguez, Y., y Cifuentes Y. (2014). Prototipo de electrocardiógrafo bipolar para uso académico. Ciencia y Poder Aéreo, 9(1), 115-123.

Robayo Alcocer, M. (2014). Marco Leonardo. Desarrollo de aplicaciones prácticas para microcontroladores ATMEL bajo la plataforma de entrenamiento arduino.

University of Chicago Medicine. (2015). Signos Vitales: Temperatura Corporal, Pulso, Frecuencia Respiratoria y Presión Sanguínea. Recuperado en diciembre de 2015, [En línea] Disponible en http://www.uchospitals.edu/online-library/content=S03963 